\title{
Assigning Quantitative Function to Post-Translational Modifications Reveals Multiple Sites of Phosphorylation That Tune Yeast Pheromone Signaling Output
}

\author{
David Pincus $^{1 \times a}$, Christopher J. Ryan ${ }^{1 \times b}$, Richard D. Smith ${ }^{2}$, Roger Brent ${ }^{19 \alpha c}$, Orna Resnekov ${ }^{1 * 9}$ \\ 1 Molecular Sciences Institute, Berkeley, California, United States of America, 2 Pacific Northwest National Laboratory, Richland, Washington, United States of America
}

\begin{abstract}
Cell signaling systems transmit information by post-translationally modifying signaling proteins, often via phosphorylation. While thousands of sites of phosphorylation have been identified in proteomic studies, the vast majority of sites have no known function. Assigning functional roles to the catalog of uncharacterized phosphorylation sites is a key research challenge. Here we present a general approach to address this challenge and apply it to a prototypical signaling pathway, the pheromone response pathway in Saccharomyces cerevisiae. The pheromone pathway includes a mitogen activated protein kinase (MAPK) cascade activated by a G-protein coupled receptor (GPCR). We used published mass spectrometrybased proteomics data to identify putative sites of phosphorylation on pheromone pathway components, and we used evolutionary conservation to assign priority to a list of candidate MAPK regulatory sites. We made targeted alterations in those sites, and measured the effects of the mutations on pheromone pathway output in single cells. Our work identified six new sites that quantitatively tuned system output. We developed simple computational models to find system architectures that recapitulated the quantitative phenotypes of the mutants. Our results identify a number of putative phosphorylation events that contribute to adjust the input-output relationship of this model eukaryotic signaling system. We believe this combined approach constitutes a general means not only to reveal modification sites required to turn a pathway on and off, but also those required for more subtle quantitative effects that tune pathway output. Our results suggest that relatively small quantitative influences from individual phosphorylation events endow signaling systems with plasticity that evolution may exploit to quantitatively tailor signaling outcomes.
\end{abstract}

Citation: Pincus D, Ryan CJ, Smith RD, Brent R, Resnekov O (2013) Assigning Quantitative Function to Post-Translational Modifications Reveals Multiple Sites of Phosphorylation That Tune Yeast Pheromone Signaling Output. PLoS ONE 8(3): e56544. doi:10.1371/journal.pone.0056544

Editor: Mohamed Ali Hakimi, Centre National de la Recherche Scientifique, France

Received October 10, 2012; Accepted January 15, 2013; Published March 12, 2013

Copyright: (C) 2013 Pincus et al. This is an open-access article distributed under the terms of the Creative Commons Attribution License, which permits unrestricted use, distribution, and reproduction in any medium, provided the original author and source are credited.

Funding: Research was supported by grant number P50 HG002370 (PI, R. Brent) from National Human Genome Research Institute. R.D. Smith acknowledges additional funding from grant number 8P41 GM103493-10 (PI, R.D. Smith) from National Institute of General Medical Sciences. The funders had no role in study design, data collection and analysis, decision to publish, or preparation of the manuscript.

Competing Interests: The authors have declared that no competing interests exist.

*E-mail: resnekov@molsci.org

9 These authors contributed equally to this work.

aa Current address: Whitehead Institute for Biomedical Research, Cambridge, Massachusetts, United States of America

ab Current address: Biophysics Graduate Group, University of California Berkeley, Berkeley, California, United States of America

ac Current address: Fred Hutchinson Cancer Research Center, Seattle, Washington, United States of America

\section{Introduction}

In response to stimuli sensed at the cell surface by receptors, eukaryotic cells propagate signal to the nucleus via intracellular signaling pathways. Such pathways inform decisions about cell fate, cell polarity, migration, cell-cycle regulation, cell proliferation and programmed cell death $[1,2,3,4,5]$. Signal transmission is often accomplished by regulated phosphorylation of protein components of signaling pathways. Phosphorylation can rapidly and reversibly modulate numerous properties of proteins including their conformation, enzymatic activity, molecular interactions, subcellular localization and surface charge $[6,7]$.

In organisms as diverse as mammals, invertebrates and yeast, researchers have used mass spectrometry based proteomics to identify thousands of sites of phosphorylation on proteins in cell signaling pathways. Some sites of phosphorylation serve as molecular switches that activate an enzyme (e.g., sites on the activation loop of kinases), determine a protein's subcellular localization (e.g., sites that exclude a transcription factor from the nucleus), or target a protein for degradation (e.g., cell-cycle controlled phospho-degrons) $[8,9,10,11]$. The roles of these types of sites are relatively straightforward to elucidate with qualitative assays since point mutants that cannot be phosphorylated either phenocopy null mutants or produce constitutive activity. However, there are many uncharacterized phosphorylation sites. We hypothesized that many of these sites exert quantitative regulatory roles in signaling pathways, the effects of which would only be revealed with quantitative assays in the context of the specific stimulus about which they convey information. We therefore developed a systematic and general approach to prioritize the study of individual phosphorylation events and their potential quantitative functions in signaling networks.

We focused on the pheromone response system in the budding yeast Saccharomyces cerevisiae - a well-developed system for studying eukaryotic cell signaling. In this system we can sensitively and 
accurately quantify pathway input, signal flow and pathway output in vivo in single cells $[12,13,14]$.

The pheromone signaling system is stimulated by binding of a 13 amino acid peptide pheromone to a 7-transmembrane spanning $G$ protein-coupled receptor (GPCR), activating a mitogen-activated protein kinase (MAPK) cascade and triggering downstream cellular events including gene transcription, cell cycle arrest, cell fusion and mating (Figure 1) [15]. Upon GPCR activation by pheromone, the $\mathrm{G} \alpha$ subunit of the heterotrimeric Gprotein binds GTP and dissociates from G $\beta \gamma$. Free G $\beta \gamma$ diffuses in the plasma membrane and provides a binding site that recruits the scaffold protein Ste5. At the plasma membrane, Ste5 recruits members of the MAPK cascade: the MAP3K Ste11, the MAP2K Ste7, and the MAPK Fus3. To initiate signaling, Stell is phosphorylated by the p21-activated kinase (PAK) Ste20, which is activated by binding to the GTP-bound form of the small GTPase Cdc42. To bridge an interaction between Stell and active Ste20, Ste 11 binds to the adaptor protein Ste50; Ste50 also binds to Cdc42, thus bringing Ste1 1 to active Ste20 [16,17]. After activation by Ste20, Ste11 phosphorylates Ste7, which in turn phosphorylates Fus3 in the presence of Ste5 [18,19]. Fus3 (an effector kinase) phosphorylates substrates in the cytoplasm to induce cell cycle arrest and polarized growth. In the nucleus, Fus3 phosphorylates the functionally redundant transcription repressors Digl and Dig2, as well as the transcription activator Ste12 to induce pheromone responsive gene expression [20,21]. Upon stimulation with pheromone, precise quantitative information about the amount of receptor occupied at the cell surface reaches the nucleus within minutes [12,13,22].

Here, we mined mass spectrometry based proteomics data to identify phosphorylation sites with no known function on components of the pheromone pathway. We then prioritized for further examination phosphopeptides that contained conserved consensus sequences (associated with MAPKs) in regions of system proteins that contained no known or predicted structural domains. Finally we combined mutations, quantitative single-cell experiments and computational modeling to define novel quantitative roles for six phosphorylation events on three pathway proteins in regulating pheromone signaling. While more detailed and mechanistic computational models of the pathway have been published before, the simple models used here are course-grained in order to be generic and broadly applicable [23,24,25].

We found that mutation of three of the six sites decreases system output, mutation of two other sites increases system output, and mutation of the final site removes a negative feedback loop that conditionally diminishes system output when signal is low. We believe that a similar combination of approaches will allow researchers to characterize functional roles for phosphorylation events that contribute to the dynamic quantitative regulation of many signaling systems.

\section{Results}

\section{Experimental overview: identifying putative sites of phosphorylation and assaying mutants for quantitative pheromone signaling phenotypes}

Ste12, Ste50 and Digl function at different points in the pheromone response system upstream and downstream of the protein kinase cascade (Figure 1). We chose 4 tryptic peptides containing reported phosphorylation sites: two on Ste12 (L398K409 and P523-R529), one on Ste50 (R200-R208), and one on Digl (V266-K282) (Table S4). We prioritized these peptides based on the following criteria: (1) Each peptide contains a residue predicted to be a MAP kinase substrate within a consensus MAP

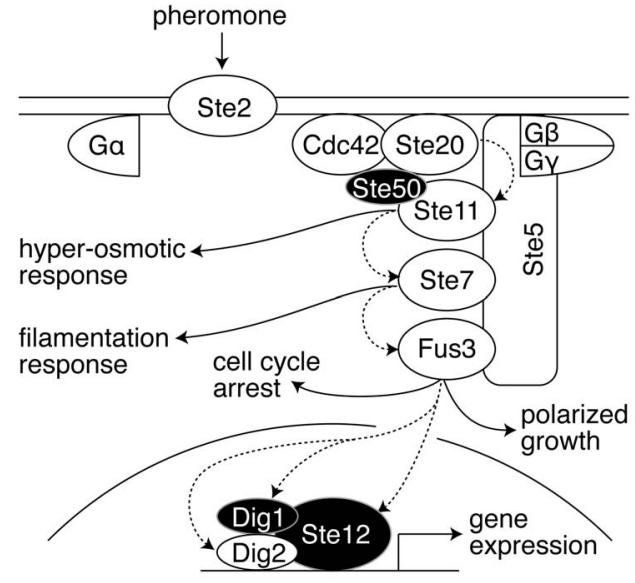

Figure 1. Pheromone induced mating pathway in yeast. Pheromone ( $\alpha$ factor) binds to a G-protein coupled receptor (Ste2). Binding promotes dissociation of the heterotrimeric $\mathrm{G}$ protein into $\mathrm{G} \alpha$ and $G \beta \gamma$. Free $G \beta \gamma$ initiates downstream signaling by forming $a$ complex with the scaffold protein Ste5 at the plasma membrane, as well as the small GTPase Cdc42, the adaptor protein Ste50 and the kinase Ste20. Ste5 further recruits the MAPK cascade members, Ste11, Ste7 and Fus3. Once this complex forms, Ste20 phosphorylates and activates Ste11; Ste11 then phosphorylates and activates Ste7; Ste7 phosphorylates and activates the MAPK Fus3. Dashed arrows indicate phosphorylation of proteins involved in the pheromone response shown here; solid arrows show connections to the high osmolarity response pathway, filamentation pathway, cell cycle control and polarized growth. Ste11 also participates in the hyper-osmotic response and the filamentation MAPK pathways, and Ste7 also participates in the filamentation pathway. Active Fus3 executes the different cellular responses to pheromone by phosphorylating and activating the transcriptional complex of Ste12, Dig1 and Dig2 to express the pheromone responsive target genes. In addition to transcriptional activation, Fus3 also arrests the cell cycle and initiates polarized growth. Figure omits other phosphorylation events and feedback. Phosphorylation events on the proteins Ste50, Dig1 and Ste12 (shaded in black) are the focus of this work.

doi:10.1371/journal.pone.0056544.g001

kinase site (S/T-P); (2) the MAPK consensus motifs are conserved from $S$. cerevisae to at least $S$. bayanus ( $\sim 20$ million years); (3) the chosen peptides lie outside of evolutionarily conserved predicted structural domains (STE on Ste12; SAM and RA domains on Ste50) $[26,27,28,29,30]$.

We made individual haploid yeast strains in which we replaced wild type STE12, STE50 or DIG1 with a mutant allele encoding the protein to be tested under the control of its native promoter at its endogenous locus (Table S1, Table S2, Table S3). We mutated single serine and/or threonine $(\mathrm{S} / \mathrm{T})$ residues as well as all $\mathrm{S} / \mathrm{T}$ residues within the regions corresponding to the observed tryptic phosphopeptides to account for potential ambiguities in identified phosphorylation sites and the possibility of multiple phosphorylated forms. To quantitatively assess pathway output in strains containing the mutated proteins, we inserted a transcriptional reporter containing the pheromone responsive $\mathrm{P}_{\mathrm{PRM} 1}$ promoter fused to a fluorescent protein (either YFP or mCherry), and used previously described fluorescence microscopy and flow cytometrybased single cell assays to measure fluorescence after treatment with different concentrations of pheromone [12,13,31]. 
Putative phosphorylation sites S400 and T525 on Ste12 are required for full induction of the pheromone response

To begin to elucidate the quantitative role of phosphorylation in the pheromone pathway, we focused on two tryptic peptides on the transcriptional activator Ste12 (Figure 2A, L398-K409 and P523-R529). Both peptides contained conserved candidate MAPK phosphorylation sites (S400 and T525) with no known roles in pheromone signaling. Phosphorylation of S400 has previously reported by several groups, and was shown to increase in abundance in cells treated with $2 \mu \mathrm{M}$ pheromone for 2 hours, but has no known function (Table S4) $[32,33,34,35]$. Since there were 4 potential phosphorylation sites on peptide L398-K409, we changed S400, S402, T405 and S406 to alanine individually, as well as in combination (4X mutant) (Figure 2A and B). In peptide P523-R529, we changed T525 to alanine (Figure 2A and B). All mutant versions of Ste12 were detectable by immunoblot at comparable levels before and after stimulation with pheromone (Figure 2B) [36].

A
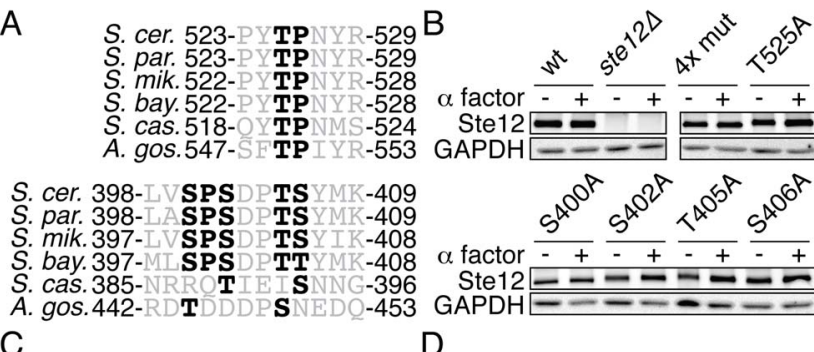

C

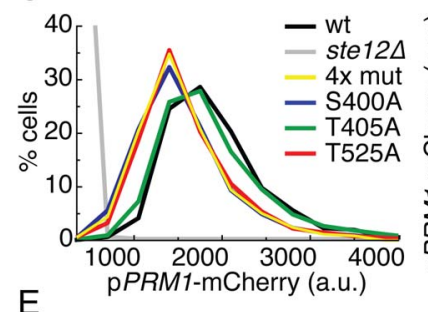

\section{D}

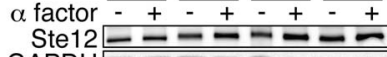

GAPDH $-1-0-1$
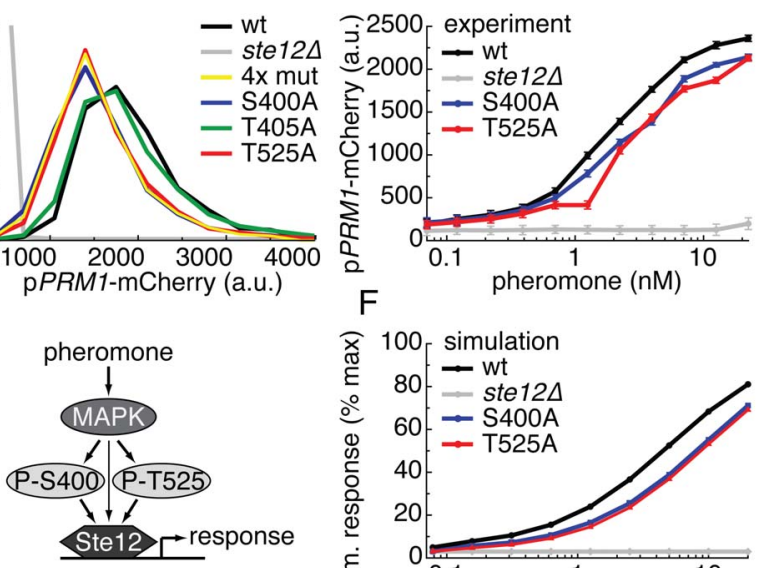

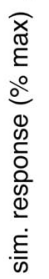

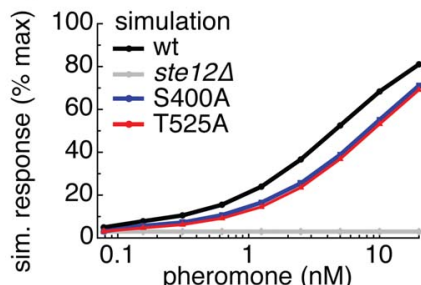

Figure 2. Two phosphorylation sites in Ste12 are required for full transcriptional induction. A. Alignment of 2 tryptic phosphopeptides across 6 yeast species. Possible phosphorylation sites (serines and threonines) and minimal MAPK consensus sequences $(\mathrm{S} / \mathrm{T}, \mathrm{P})$ are in bold. B. Western blots of lysate from yeast strains containing the indicated wild type or mutant Ste12, treated or not treated with $1 \mu \mathrm{M}$ alpha factor for 15 minutes probed with anti-Ste12 and anti-GAPDH. $4 x$ mut is Ste12 $2^{\text {S400A,S402A,T405A,S406A }}$. C. Histograms of the single cell $(n>$ 2000 cells) pheromone response measured by pPRM1-mCherry fluorescence. Cells were treated with $4 \mathrm{nM}$ alpha factor for $3 \mathrm{hrs}$ followed by cycloheximide for $2 \mathrm{hrs}$, and measured by flow cytometry. D. Dose responses of cells treated with 12 concentrations of pheromone as described in C. Figure plots means of the unimodal distributions. Error bars depict the standard error of the mean. E. Cartoon model of the function of the phosphorylation of S400 and T525 F. Simulation of the ODE model across a dose response of pheromone. doi:10.1371/journal.pone.0056544.g002
To quantify pheromone pathway output in these strains we used flow cytometry to measure signal from a fluorescent reporter gene fused to a pheromone responsive promoter ( $\mathrm{P}_{P R M 1}$-mCherry) $[12,13,37]$. After treatment with $4 \mathrm{nM} \alpha$-factor (pheromone), the $4 \mathrm{X}$ mutant strain, the Ste $12^{\mathrm{S} 400 \mathrm{~A}}$ strain, and the Ste $12^{\mathrm{T} 525 \mathrm{~A}}$ strain all displayed statistically significant $\sim 25 \%$ decreases in fluorescence compared to the wild type reference strain (Figure $2 \mathrm{C}$, Table S5). The fluorescence in strains bearing Ste $12^{\mathrm{S} 402 \mathrm{~A}}$, Ste $12^{\mathrm{T} 405 \mathrm{~A}}$, and Ste $12^{\mathrm{S} 406 \mathrm{~A}}$ was indistinguishable from the wild type reference strain (Figure 2C, Figure S1). The diminished output of the Ste $12^{\mathrm{S} 400 \mathrm{~A}}$ and Ste $12^{\mathrm{T} 525 \mathrm{~A}}$ strains persisted across a wide range of $\alpha$-factor doses (Figure 2D). These results are consistent with the notion that phosphorylation of S400 and T525 is required to fully activate Ste 12 .

A simple computational model of phospho-regulation of Ste12 S400 and T525 recapitulates the quantitative mutant phenotypes

To explore how phosphorylation of S400 and T525 regulate pheromone signaling, we developed a simple mathematical model of the pheromone pathway (Figure 2E, Figure S2, Figure S3). This model omitted many of the interactions and molecular states involved in pheromone signaling, and thus minimized the number of unmeasured parameters that are commonly needed to construct chemical reaction models of biological systems [38]. Our mass action based model consisted of a set of six differential equations. Each differential equation described the rate of change in activity of a single species, and each species has parameters associated with it that describe basal synthesis rate, degradation rate, and the strength of its activity. Structurally, the models represented phospho-S400 and phospho-T525 as distinct species, and activation of Ste12 depended on their "concentration" (Figure S2). We simulated site mutants by setting the concentration of the phosphorylated species to 0 while keeping other parameter values constant. This approach facilitated simulation and exploration of different possible regulatory architectures in both reference and site mutant strains.

We based the model on two assumptions. First, since in the Ste12 sequence both S400 and T525 precede proline residues (the minimum consensus requirement for MAPK substrates), we assumed that the MAPK Fus3 phosphorylates these residues $[39,40]$. Second, we assumed that phosphorylation of S400 and T525 and their effects on Ste12 were independent of each other. To explore the effects of the site mutants, we simulated both "wild type" and "mutant" models across the broad range of pheromone inputs for which we had experimental data (Figure 2F). We selected generic parameter sets and analyzed the sensitivity of the model to each parameter (Figure S3). The simulated reference and mutant circuits recapitulated experimentally observed diminution of pathway output across a broad range of parameters. Thus, the data are consistent with a model in which phosphorylation of S400 and T525 increase the gain of the system.

\section{Ste50 S202 inhibits signaling at low concentrations of pheromone}

We next extended our interrogation of the quantitative phospho-regulation of the pheromone pathway to the adaptor protein Ste50 that lies upstream of the MAPK cascade. We focused our attention on the phosphorylated tryptic peptide R200R208 (Table S4) that contains a conserved putative MAPK phosphorylation site at S202 with no known role in pheromone signaling (Figure 3A) [32,33,34]. We changed S202 and T205 to alanine individually, as well as in combination (2X mutant) (Figure 
A

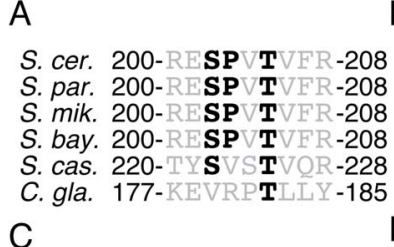

$\mathrm{C}$

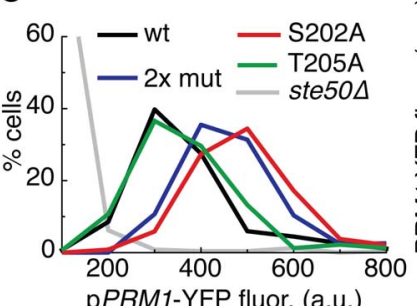

E
B

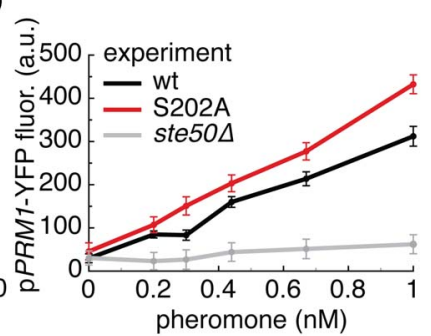

$\mathrm{F}$

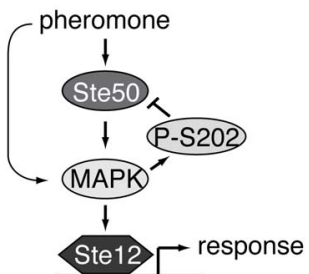

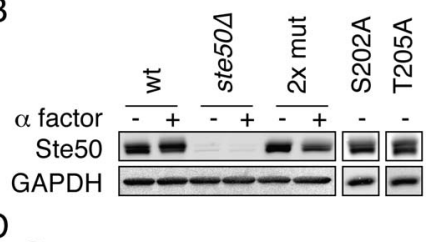

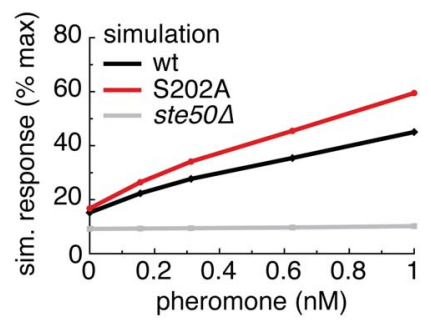

Figure 3. Phosphorylation of S202 on Ste50 inhibits pathway activity at low doses of pheromone. A. Alignment of a Ste50 tryptic phospho-peptide across 6 yeast species. Possible phosphorylation sites (serines and threonines) and minimal MAPK consensus sequences ( $\mathrm{S} /$ $\mathrm{T}, \mathrm{P})$ are in bold. This peptide is not within either the SAM or the RA domains. B. Western blots of lysate from yeast strains containing the indicated wild type or mutant Ste50 that had been in the absence or presence of $1 \mu \mathrm{M}$ alpha factor for 15 minutes probed with anti-Ste50 and anti-GAPDH. C. Histograms of the single cell pheromone response measured by pPRM1-YFP fluorescence. Cells ( $\mathrm{n}>300$ cells) were treated with $1 \mathrm{nM}$ alpha factor for $3 \mathrm{hrs}$ followed by cycloheximide for $2 \mathrm{hrs}$, imaged by fluorescent microscopy, and quantified with Cell-ID. D. Dose responses of cells treated with 5 concentrations of pheromone as described in C. The means of the unimodal distributions are plotted. Error bars depict the standard error of the mean. E. Cartoon model of the function of the phosphorylation of S202. F. Simulation of the ODE model across a dose response of pheromone.

doi:10.1371/journal.pone.0056544.g003

$3 \mathrm{~A}$ and B). Mutant versions of Ste50 were detectable by immunoblot at comparable levels (Figure 3B) [36].

To quantify pheromone pathway output in strains containing the mutated proteins, we used microscopy coupled to the Cell-ID image analysis software to measure fluorescence from $\mathrm{P}_{P R M 1}$-YFP $[12,13,41]$. The strain expressing Ste $50^{\mathrm{S} 202 \mathrm{~A}}$ and the $2 \mathrm{X}$ mutant strain showed increased fluorescence at a low dose $(1 \mathrm{nM})$ of pheromone compared to the wild type reference, while the Ste $50^{\text {T205A }}$ strain was indistinguishable from wild type (Figure 3C). No phenotype was observed at higher doses of pheromone. To better resolve system output at low pheromone inputs we used strains that carried a mutant cyclin-dependent kinase (Gdc28-as2) sensitive to the chemical inhibitor 1-NM-PP1 [42]. In these cells, addition of 1-NM-PP1 arrests progression through the cell cycle, thus removing a major contribution of cell-to-cell variation in pheromone system output [12]. After treatment with 1-NM-PP1, Ste $50^{\mathrm{S} 202 \mathrm{~A}}$ and the $2 \mathrm{X}$ mutant displayed increased output when stimulated with pheromone across a dose range of $0.2-1 \mathrm{nM}$ compared to the wild type reference and the Ste $50^{\text {T205A }}$ strain (Figure 3D and Figure S4). These data suggest that phosphory- lation of S202 is required to prevent hyper-activation of the pathway at low doses of pheromone.

Computational modeling supports a negative feedback mechanism via phosphorylation of Ste50 S202

To formalize our notion that phospho-S202 prevents hyperactivation of the pheromone pathway we constructed a mathematical model. Based on the facts that Ste 50 acts upstream of the MAPK cascade and S202 is a predicted MAPK substrate, we structured the model such that phospho-S202 acts as a negative feedback loop (Figure 3E, Figure S5). The model consists of five differential equations describing the rates of change of the species, including phospho-S202. Simulation of the model across the experimental pheromone dose response range recapitulated the sensitization in the S202A mutant over a wide range of parameter values (Figure 3F and S5). Our results are consistent with the idea that phosphorylation of S202 on Ste50 defines a negative feedback loop that dampens pheromone pathway output and prevents hyper-activation of the pathway at low doses of pheromone.

\section{Dig1 is a co-activator of transcription in the presence of} CFP-Ste12

We concluded our initial examination of the quantitative phospho-regulation of the pheromone pathway by focusing on Dig 1, a functionally redundant transcriptional repressor of Ste12 [43]. Though Digl is phosphorylated at $\geq 18$ sites, no site has a known function [32,33,34,35]. We focused on the multiply phosphorylated tryptic peptide V266-K282. V266-K282 contains a putative MAPK phosphorylation site at S272 and a predicted nucleotide-binding motif (Figure 4A).

Functionally redundant proteins present a distinct challenge to studying the functional consequences of individual phosphorylation events on the quantitative modulation of signal propagation, since a deletion of one of the two functionally redundant genes has no phenotype. One proven genetic approach is to delete the second functionally redundant gene (here it would be DIG2), and examine the effect of mutations in the first protein in the deleted strain background. However, this approach is not suitable for all experimental systems and may result in pleiotropic effects due to a lack of complete redundancy. Here, we chose to investigate the suitability of using a sensitized genetic background (a background that reveals a phenotype that would not be clear in a wild type background). For this purpose we turned to a strain that was constructed to measure the Digl-Ste12 interaction in vivo in single cells using FRET (fluorescence resonance energy transfer) [13]. The strain contained YFP-tagged Digl that can be co-expressed with CFP-tagged Ste12, and we additionally engineered this strain to contain the $\mathrm{P}_{P R M 1}$-mCherry reporter to simultaneously measure pheromone pathway output.

Since the peptide V266-K282 contained four serine and two threonine residues, we chose to introduce two separate $3 \mathrm{X}$ DiglYFP mutant constructs (S269A, S272A, S275A and T277A, S279A, T280A) as well as individual Digl-YFP point mutants into the DIG1 chromosomal locus under the control of the native DIG1 promoter. We verified the comparable expression level of the mutant proteins in each resulting strain by immunoblot and their nuclear localization by fluorescence microscopy (Figure 4B and Figure S6) [36].

The reference strain containing CFP-Ste12 and Digl-YFP showed robust pheromone-dependent transcriptional induction as measured by quantitative fluorescence microscopy (Figure 4G). Surprisingly, however, CFP-Ste12 showed a severely impaired transcriptional response in a diglã background (Figure 4C). In the 
A

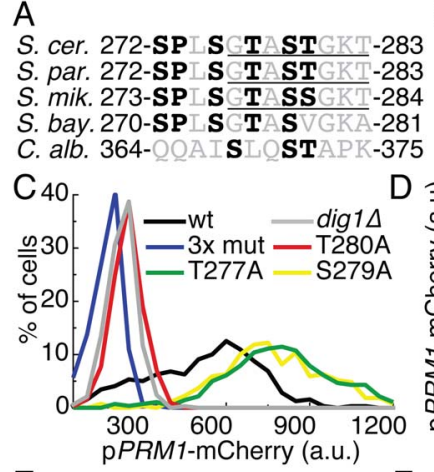

B

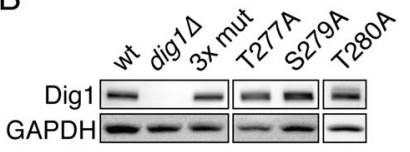

E

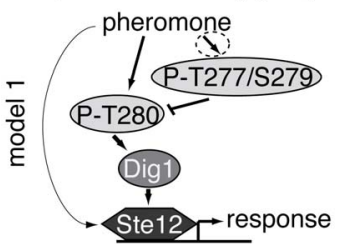

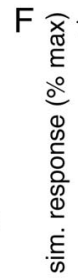
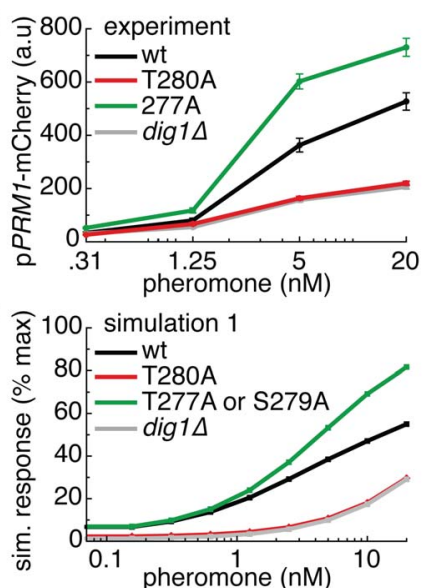

G

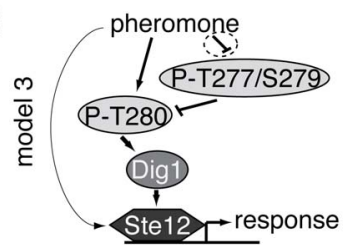

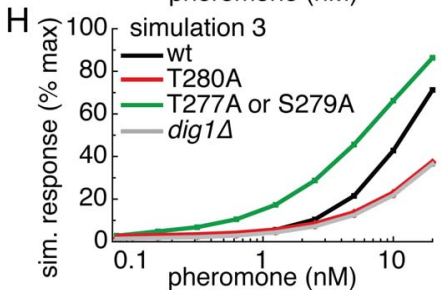

Figure 4. Opposing phenotypes in Dig1 phospho-mutants. A. Alignment of an 12-amino acid sequence, part of a tryptic phosphopeptide, in 5 yeast species. Possible phosphorylation sites (serines and threonines) and minimal MAPK consensus sequences (S/T,P) are in bold. The "TAST" sequence in S. cer. Dig1 falls in the variable region of a bioinformatically predicted ATP/GTP binding motif ( $p$-loop, underlined). B. Western blots of lysate from yeast strains containing the indicated alleles of Dig1-YFP probed with rabbit anti-Dig1 and anti-GAPDH. $3 \times$ mut is Dig1 T277A,S279A,T280A. C. Histograms of the single cell pheromone response measured by $\mathrm{p} P R M 1$-mCherry fluorescence. Cells ( $\mathrm{n}>500$ cells) were treated with $20 \mathrm{nM}$ alpha factor for $3 \mathrm{hrs}$ followed by cycloheximide for $2 \mathrm{hrs}$, imaged by fluorescent microscopy, and quantified with Cell-ID. D. Dose responses of cells treated with the indicated concentrations of pheromone as described in C. The means of the unimodal distributions are plotted. Error bars depict the standard error of the mean. E. Cartoon of model 1, in which pheromone induces phosphorylation of all residues. F. Simulation of model 1 across a dose response of pheromone. G. Cartoon of model 3, in which pheromone induces the dephosphorylation of T277/S279 and the phosphorylation of T280. H. Simulation of model 3 across a dose response of pheromone.

doi:10.1371/journal.pone.0056544.g004

absence of the CFP tag, untagged Ste12 is sufficient to activate pheromone target genes in a diglã background [44]. However, when tagged on its N-terminus, CFP-Ste 12 required Digl as a coactivator. While normally Digl acts as a repressor to Ste12, by Nterminally tagging Ste 12 with a fluorescent protein, we effectively reversed the regulatory relationship of Ste12 with its binding partner Digl. This novel reliance on Digl for pheromone-induced transcriptional activation is consistent with recent work showing that Dig1 and Dig2 play positive roles in the pheromone response [45]. The sensitized CFP-Ste12, Digl-YFP background allowed us to monitor the effect of mutations of putative phosphorylation sites on Digl on pathway output, in the presence of Dig2.

Closely situated Dig1 mutations have opposing effects on pathway output

We took advantage of this increased reliance on Digl for pheromone output to explore novel signaling phenotypes of phosphorylation site mutants. We first measured $\mathrm{P}_{P R M I}$-mCherry fluorescence in cells treated with a high dose of $20 \mathrm{nM} \alpha$-factor. Output from the first 3X mutant strain (Digl-YFP $269 \mathrm{~A}$, S272A, S275A) was indistinguishable from reference cells (Figure S7). By contrast, output from the second 3X mutant (Dig1-YFP ${ }^{\mathrm{T} 277 \mathrm{~A}, \mathrm{~S} 279 \mathrm{~A}, \mathrm{~T} 280 \mathrm{~A}}$ ) was severely diminished, similar to the diglã strain (Figure 4C).

Strains that carried individual mutations of T277, S279, or T280 each showed altered pathway output compared to wild type Digl-YFP. Output from Dig $1^{\text {T280A }}$ was diminished compared to the reference strain, similar to the $3 \mathrm{X}$ mutant strain (Figure 4C). However, in the T277A and S279A strains, output was significantly higher than the reference strain (Figure 4C). These phenotypes persisted across a dose response (Figure 4D). Surprisingly site-specific mutation of closely situated residues within Digl-YFP had opposite effects on system output.

\section{Modeling complex Dig1 phospho-regulation constrains possible pathway architectures}

To develop a better understanding of the opposing roles of the Digl mutations in the context of the novel co-activator role of Digl-YFP, we again turned to computational modeling. The Dig 1 model treated Digl as an activator of Ste12, and incorporated the constraint that the T280A mutation was dominant over the T277A and S279A mutations since the T280A phenotype masked the T277A and S279A phenotypes in the 3X mutant. Digl residues T277, S279 and T280 are not likely to be MAPK substrates. Thus, developing a model for Dig 1 required exploration of several possible pathway architectures.

We constructed and studied four different models: 1) in which all phosphorylation was induced by pheromone; 2) in which all sites were initially phosphorylated and dephosphorylation was pheromone-dependent; 3) in which T280 was phosphorylated in response to pheromone while T277/S279 were dephosphorylated in response to pheromone; and 4) in which all phosphorylation was constitutive and independent of pheromone (Figure 4E, 4G, and Figure S8). We studied the behavior of the four models as a function of pheromone concentration. We were unable to recapitulate the opposing single-mutant phenotypes with either a constitutive phosphorylation model (model 2) or a model in which all sites were dephosphorylated after pheromone induction (model 4) (Figure S8). However, both the model in which all the sites were phosphorylated in response to pheromone (model 1), and the model in which T280 was phosphorylated in response to pheromone while T277/S279 were dephosphorylated in response to pheromone (model 3), satisfied the experimental constraints (Figure $4 \mathrm{~F}$ and $4 \mathrm{H}$ ). While model 1 fit the data over a larger range of parameters, model 3 better reproduced the hypersensitivity of T277A at low doses of pheromone (Figure 4F, 4H, Figure S9, and Figure S10). Thus, for these models to recapitulate the experimental results, T280 must be phosphorylated in response to pheromone, while at the same time T277/S279 must either be phosphorylated or dephosphorylated in response to pheromone.

\section{Discussion}

While many sites of phosphorylation have been mapped in proteomic studies from mammals, invertebrates and yeast, the vast majority of sites have no known function. We hypothesized that many of these sites are likely to exert dynamic regulatory roles in signaling pathways, the effects of which can only be revealed with 
quantitative assays in the context of the specific stimulus about which they convey information. We therefore developed a systematic, general approach to prioritize the study of individual phosphorylation events and their potential functions in signaling networks, and demonstrated the utility of our approach on components of the pheromone response system in the budding yeast Saccharomyces cerevisiae.

Since the approach described here requires targeted mutagenesis of genomic DNA, which is straightforward to accomplish in genetically tractable model organisms like yeast, we acknowledge that this methodology is more challenging to implement in other organisms. However with recent advances in tools like zinc finger nucleases and TALENs it will become increasingly possible to target mutations in many diverse organisms and cell types [46]. We believe that the approach that we have employed here will soon be applicable to, e.g., iPS cells.

We focused on three non-kinase proteins with distinct signaling roles upstream and downstream of the protein kinase cascade (Figure 1): 1) Ste12, a transcription factor activated by the MAP kinase cascade that induces genes involved in mating, 2) Ste50, an adaptor protein that acts upstream of the MAP kinase cascade to link the $G$ protein-associated rho-like GTPase (Cdc42)-PAK kinase (Ste20) complex to the MAP3K (Ste11) and, 3) Digl, a protein involved in regulating the activity of Ste12. Using quantitative single cell assays we showed that 6 phosphorylation sites on 4 separate motifs in 3 signaling components (Ste12, Ste50 \& Dig1) quantitatively affected pheromone pathway output. While the most parsimonious interpretation of the quantitative phenotypes we observed is that the changes in pathway output are the direct consequence of the inability of the mutated residue to be phosphorylated, we have not ruled out that the altered pathway output we observed is due to structural changes unrelated to phosphorylation and/or altered protein/protein interactions caused by the alanine substitutions.

In strains expressing a protein with phosphorylation site point mutations, no cell will have the mutated site phosphorylated. In the reference strain in any given single cell, the fraction of the population of molecules of the protein with the site phosphorylated is unknown. We can thus expect that the effect of any phosphorylation site on signaling pathway output will be incompletely penetrant. The quantitative phenotypes we measured in the mutant strains - measurements made comparing the population of mutant cells to the heterogenous reference cells may therefore underestimate the effect that these phosphorylation sites exert on the activity of the proteins they modify.

On Ste12, we identified 2 putative phosphorylation sites, S400 and $\mathrm{T} 525$, that each contribute $\sim 25 \%$ to the transcriptional activity of Ste 12 across a dose response of pheromone (Figure 2D). Based on neighboring sequence context, these sites are likely to be MAPK targets and we demonstrated that they both contribute to the appropriate transcriptional output to a given dose of pheromone. The conservation of these sites among closely related yeast species and the quantitative agreement of the computational model with the experimental results lend credence to this notion (Figure 2). While the $25 \%$ gain in activity afforded by these sites is unlikely to be absolutely required for mating in laboratory settings, such a quantitative increase in output may have conferred competitive fitness over evolutionary timescales.

The adaptor protein Ste50 links the MAP3K Stell to active Cdc42 and Ste20, thereby localizing Ste11 to its upstream activators at the plasma membrane. Stell then signals through different MAPK cascades via its association with different scaffold proteins: Ste5 directs signaling to the pheromone pathway, while Pbs2 directs signaling to the hyper-osmotic stress pathway. Several groups have suggested that phosphorylation of Ste50, possibly on S202, may help determine how much signal from active Ste11 goes to the pheromone pathway, and how much goes to the hyperosmotic stress pathway [47,48,49]. Mechanistically, phosphorylation of Ste50 on S202 may influence pathway choice by regulating protein-protein interactions with membrane anchoring factors that associate with the pathway-specific scaffolds $[47,49]$. Here we propose that phosphorylation of S202 on Ste50 anchors a negative feedback loop that inhibits pheromone pathway output in response to low doses of pheromone (Figure 3). Thus, phosphorylation of S202 may serve to dampen the threshold required to activate a full-fledged pheromone response. This negative feedback loop could be relevant when mating partners are scarce and commitment to the mating program would waste resources, and also when both pheromone and high osmolarity are present to ensure that enough signaling bandwidth is available to trigger the response to osmotic stress.

For the redundant repressor protein Digl, we utilized a sensitized genetic background in which CFP-tagged Ste 12 requires Digl for its full activity to increase the likelihood of revealing functional roles for Digl mutants without having to delete the paralogous repressor Dig2. We identified 3 putative sites on Digl that alter the pheromone response. T280 is required for Diglmediated Ste12 activity: cells expressing Digl-YFP ${ }^{\mathrm{T} 280 \mathrm{~A}}$ displayed a severely diminished transcriptional response, phenocopying the full deletion of Dig1. Strikingly, the closely situated residues T277 and S279 had the opposite effect in that they inhibit signal output: mutation of either residue leads to an increased pheromone response. Since these sites lack consensus MAPK sequence context, the mechanistic and architectural details of how they might exert their effects could be constrained through computational modeling, but not determined unequivocally. Similarly, since in this sensitized strain background, CFP-Ste12 required Digl-YFP for full activity, the significance of the phenotypes of the Digl mutations for the native pheromone pathway remains to be determined. However, our current findings that closely situated mutations exert opposite effects are sufficient to suggest that multiple layers of post-translational regulation can be superimposed to fine-tune quantitative signal output.

Our results indicate that many putative sites of phosphorylation contribute to and adjust the input-output relationship of this model eukaryotic signaling system. We propose that multiple small influences of such individual phosphorylation events can endow signaling systems with plasticity and evolvability. Consistent with this view, in the motifs that we studied on Ste12 (S400, T525) and Ste50 (S202), the presence of a minimum consensus requirement for a MAPK substrate (serine or threonine preceded by a proline residue) arose relatively recently and is conserved in closely related yeast species but not in more distantly related yeast species (Figures 2A and 3A). In Digl, where residues T277, S279 and T280 are not likely to be MAPK substrates, the presence of serine or threonine residues at similar positions in orthologs is conserved in closely related yeast species but not in more distantly related yeast species (Figure 4A). In all cases, the putative phosphorylation events that we studied fall in regions predicted to be unstructured in Ste12, Ste50 and Digl. Such unstructured regions are well suited to accommodate amino acid changes to generate new sites for post-translational events like phosphorylation [50,51,52,53,54]. This plasticity and evolvability may be advantageous for finetuning the input/output relationship of the pathway. By contrast, the core activation mechanism of the pathway is highly conserved during evolution. Activation of the MAPK Fus3 is governed by a dual phosphorylation event (T180, Y182) and mutation of either site results in a complete loss of function [9]. This dual 
phosphorylation motif in the kinase activation loop is conserved in MAPKs all the way from yeast to mammalian MAPKs such as Erk1 and Erk2 [9].

In conclusion, we suggest that new layers of post-translational regulation can be gained and lost to rapidly adapt quantitative system output in the face of changing selective pressure without compromising the core structural and functional integrity of key signaling proteins. We note that this idea suggests means to systematically design and alter signaling pathway components to introduce novel regulatory loops or sever existing ones, and to confer new regulatory properties to pathway specific kinases and phosphatases. An approach based on iterating the design and construction of such re-engineered signaling pathways guided by quantitative experimentation and interpreted via appropriate models, should facilitate design-based alteration of signaling systems to bring about desired cellular behaviors.

\section{Materials and Methods}

\section{Cell growth and reagents}

Routine growth of yeast strains was performed as described (1). Strains were grown on YAPD plates (YPD with supplemental adenine) and/or YAPD liquid (YPD with supplemental adenine) media. Yeast deletions were selected on YAPD/HygB or YAPD/ Kan plates. Mutant strains were grown on SD plates and/or SD liquid media (yeast nitrogen base, $2 \%$ glucose, with appropriate selection for auxotrophic markers).

\section{Pheromone synthesis}

Alpha factor (or pheromone; Trp-His-Trp-Leu-Gln-Leu-LysPro-Gly-Gln-Pro-Met-Tyr) was ordered from and synthesized at the W.M. Keck Foundation Biotechnology Resource Laboratory (Yale University). We made a $1 \mathrm{mM}$ stock solution and stored it in aliquots at $-80^{\circ} \mathrm{C}$.

\section{Pheromone treatment}

For large-scale cell growth cells were treated with $1 \mu \mathrm{M}$ pheromone for the time indicated. For both microscopic and flow cytometric studies cells were treated with 1-20 nM pheromone as indicated for the described time.

Pheromone sensitivity assays. We qualitatively tested the pheromone sensitivity of all reference strains, deletion strains and phosphorylation site mutants. We prepared YAPD plates with 10fold dilutions of pheromone, ranging from $1 \mu \mathrm{M}$ to $1 \mathrm{nM}$. We diluted saturated overnight cultures of strains to $\mathrm{OD}_{600}=1.0$. On each of the four plates we spotted 10, 100 and 1000 cells of each strain and incubated the plates $30^{\circ} \mathrm{C}$ for $20 \mathrm{hrs}$, following which the growth of cells was recorded.

\section{Antibodies}

We used custom rabbit polyclonal antibodies against pheromone pathway proteins (2). We monitored phosphorylation of the MAPKs (Fus3 and Kss1) in populations of cells by Western blot analysis with rabbit anti-phospho-p44/42 antibodies (Cell Signaling Technologies, Beverly, MA). We monitored the appearance of fluorescent protein fusion proteins by Western blot analysis using mouse monoclonal anti-GFP antibodies (JL-8) (BD Biosciences, Palo Alto, CA). We verified the relative abundance of cell extract per gel lane by Western blot analysis using a mouse monoclonal antibody against the reference protein, GAPDH (Abcam, Cambridge MA)._We used one of two fluorescently-labeled secondary antibodies to visualize all results on Westerns (Alexa Fluor ${ }^{\circledR} 680$ series, Invitrogen Co., Carlsbad, CA; IRDye 800 series, Rockland Immunochemicals Inc., Gilbertsville, PA).

\section{Construction of deletion strains}

We created yeast strains lacking the ORFs STE12, DIG1 and STE50. STE12: We deleted STE12 from ACLY379 using PCRmediated one-step replacement (1) with the pFA6a-kanMX6 template (3) and primer pair STE12KOf/STE12KOr, creating D12. We verified the deletion by PCR and Western blot analysis. Subsequently, we replaced the PRM1 ORF in D12 with a PCR product containing the mCHERRY coding sequence, the ADH1 terminator and the $h p h$ gene from pAG32-hphMX6 (4), creating DPY112. DIG1: We deleted DIG1 by one-step excision of ura3marked DIG1- YFP from RGY1130 using 5-FOA (1) to select for excision products, creating RCY2005. We verified the deletion using epifluorescent microscopy and Western blot analysis. Subsequently, we replaced the PRM1 ORF in RCY2005 with a PCR product containing the mCHERRY coding sequence, the $A D H 1$ terminator and the $h p h$ gene from pAG32-hphMX6 (4) creating RGY2005pch. STE50: We deleted STE50 from TCY3154 using PCR-mediated one-step replacement (1) with the pAG32-hphMX6 (4) template and primer pair STE50KOf/ STE50KOr, creating DPY250. We verified the deletion by PCR and Western blot analysis.

\section{Construction of strains containing mutant proteins}

We created yeast strains with mutant alleles of STE12, DIG1-YFP and STE50. To make the mutant STE12 strains, we amplified the wild type STE12 gene (including 968 bp of endogenous promoter, the ORF and 634 bp of endogenous terminator) from W303a genomic DNA and cloned this PCR product into pRS406 at XhoI and EcoRI sites, creating pSTE12-406. We performed site-directed mutagenesis with the GeneTailor ${ }^{\mathrm{TM}}$ site directed mutagenesis kit (Invitrogen Co., Carlsbad, CA) using the following primer pairs: STE12M3f/STE12M3r, STE12-s400a-f/STE12-s400a-r, STE12s402a-f/STE12-s402a-r, STE12-t405a-f/STE12-t405a-r, STE12s406a-f/STE12-s406a-r and STE12M4f/STE12M4r and methylated pSTE12-406 as the template, creating pSTE12m3-406, pSTE12s400a-406, pSTE12s402a-406, pSTE12t405a-406, pSTE12s406a-406, and pSTE12m4-406. The sequences of the mutated plasmids were verified (MWG Biotech, High Point, NG). We linearized the mutant plasmids in the STE12 promoter region with BsiWI (New England Biolabs, Beverly, MA), and individually transformed them into DPY112 and plated on SD plates lacking uracil, creating DPY1203, CRY1004, CRY1005, CRY1006, CRY 1007 and DPY1204. We verified the presence of and normal abundance of Ste 12 ${ }^{\mathrm{S} 400 \mathrm{~A}, \mathrm{~S} 402 \mathrm{~A}, \mathrm{~T} 405 \mathrm{~A}, \mathrm{~S} 406 \mathrm{~A}}, \mathrm{Ste} 12^{\mathrm{S} 400 \mathrm{~A}}, \mathrm{Ste} 12^{\mathrm{S} 402 \mathrm{~A}}$, Ste $12^{\mathrm{T} 405 \mathrm{~A}}$, Ste $12^{\mathrm{S} 406 \mathrm{~A}}$, and Ste $12^{\mathrm{T} 525 \mathrm{~A}}$ proteins using Western blot analysis and the absence of the Ste12 protein in the Ste12D reference strain.

To make the DIG1-YFP mutant strains, we performed site-directed mutagenesis and sent for sequencing as above, using primer pairs DIG1M1f/DIG1M1r, DIG1M3f/DIG1M3r, Digl-t277a-f/Dig1t277a-r, Digl-s279a-f/Digl-s279a-r, and Dig1-t280a-f/Digl-t280a-r and methylated pDIG1YFP-406 (3) template, creating pDIG1m1YFP406, pDIG1m3YFP-406, pDIG1t277aYFP-406, pDIG1s279aYFP406, and pDIG1t280aYFP-406. We linearized the mutant plasmids in the DIG1 promoter region with BstEII (New England Biolabs, Beverly, MA), individually transformed them into RCY2005pch and plated on SD plates lacking uracil, creating DPY1001, DPY1003, TCY3328, TCY3329, and TCY3330. We verified both the presence and normal

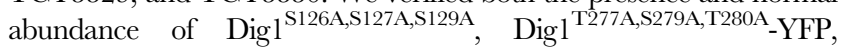
Digl ${ }^{\text {T277A }}-$ YFP, Digl ${ }^{\text {S279A }}-$ YFP, and Digl ${ }^{\text {T280A }}-$ YFP proteins by Western blot analysis and the absence of the Digl protein in the Dig1D reference strain. We additionally verified that the Dig1-YFP proteins localized to the nucleus using epifluorescent microscopy. 
To make the STE50 mutant strains, we amplified the wild type STE50 gene (including $76 \mathrm{bp}$ of endogenous promoter, the ORF and $384 \mathrm{bp}$ of endogenous terminator) from W303 genomic DNA and cloned the PCR product into pRS406 at the XhoI site, creating pSTE50-406. We performed site-directed mutagenesis and sent for sequencing as above, using primer pairs STE50Mlf/ STE50M1r, STE50-s202a-f/STE50-s202a-r, and STE50-t205a-f/ STE50-t205a-r and methylated pSTE50-406 as the template, creating pSTE50m1-406, pSTE50s202a-406, and pSTE50t205a406. We linearized the mutant plasmids in the STE50 promoter region with PshAI (New England Biolabs, Beverly, MA), transformed into DPY250 and plated on SD plates lacking tryptophan and uracil, creating DPY5001, TCY3344.2, and TCY3345.2. We verified the presence and normal abundance of Ste $50^{\mathrm{S} 202 \mathrm{~A}, \mathrm{~T} 205 \mathrm{~A}}$, Ste $50^{\mathrm{S} 202 \mathrm{~A}}$, and Ste $50^{\mathrm{T} 205 \mathrm{~A}}$ proteins by Western blot analysis and the absence of the Ste 50 protein in the Ste50D reference strain.

\section{Fluorescent transcription assays of phosphorylation mutant strains}

We quantitatively tested the pheromone response of all strains by measuring FP fluorescence in single cells as a surrogate for transcriptional output from a pheromone responsive promoter $\left(\mathrm{P}_{\mathrm{PRM} 1}-\mathrm{YFP}\right)$. Starting from single colonies on YAPD plates, we inoculated $4 \mathrm{~mL}$ cultures in YAPD. We monitored growth by $\mathrm{A}_{600}$ and diluted into $4 \mathrm{~mL} \mathrm{SDC}$ at the end of the day to obtain mid-log phase cultures in the morning. We diluted cultures to $\mathrm{OD}_{600}=0.2$ in YAPD and grew to $\mathrm{OD}_{600}=0.8$. We centrifuged $1.5 \mathrm{~mL}$ cells for $30 \mathrm{sec}$ at $15,000 \mathrm{rpm}$, resuspended in $1.5 \mathrm{~mL}$ SDC with $40 \mu \mathrm{g} / \mathrm{mL}$ casein (from DIG nucleic acid detection kit, Roche Diagnostics Corporation, Indianapolis, IN) and sonicated the cells to disperse clumps. We pipetted $500 \mu \mathrm{L}$ of each strain into a 96 well deep well plate for the untreated (no pheromone) samples and added $50 \mu \mathrm{L} 50 \mu \mathrm{g} / \mathrm{mL}$ cycloheximide (Calbiochem (EMD), San Diego, CA). We pipetted $495 \mu \mathrm{L}$ of each strain into the same 96 well deep well plate and added $5 \mu \mathrm{L}$ of $100 \mu \mathrm{M}$ pheromone in $\mathrm{SDC} /$ casein. We incubated the plate at $30^{\circ} \mathrm{C}$ for 15 minutes with shaking (300 rpm) and stopped the experiment with $50 \mu \mathrm{L} 50 \mu \mathrm{g} /$ $\mathrm{mL}$ cycloheximide in SDC/casein (Calbiochem (EMD), San Diego, CA). We incubated an additional $2.5 \mathrm{hrs}$ at $30^{\circ} \mathrm{C}$, shaking at $300 \mathrm{rpm}$, to allow for fluorophore maturation. We sonicated the cells again, diluted 1:20 in SDC/casein/cycloheximide, pipetted $200 \mu \mathrm{L}$ into 96 well glass bottom plates and let the cells settle for 10 minutes. We performed optical microscopic cytometry and image capture as described elsewhere $(5,6)$ and image analysis, and data processing using the open source software packages CellID and $\operatorname{PAW}(7,8)$.

We compared pathway output in ACLY379pch, DPY112, DPY1203, GRY1004, CRY1005, CRY1006, and CRY1007. Starting from single colonies, we inoculated $4 \mathrm{~mL}$ cultures in SDC (ACLY379pch and DPY112) or SD/-U (DPY1203, CRY1004, CRY1005, CRY1006, and CRY1007) and grew over the course of the day to obtain log phase cells. We diluted overnight $4 \mathrm{~mL}$ cultures in the same media to obtain mid-log phase cells in the morning, at which point we adjusted to $\mathrm{OD}_{600}=0.25$ and allowed the cells to grow for one generation (90 minutes). We prepared a 4-fold dilution series of pheromone in $\mathrm{SDC} /$ casein ranging from $20 \mathrm{nM}-0.31 \mathrm{nM}$. We aliquoted $7 \times 0.5 \mathrm{~mL}$ of each dilution into a 96 -well deep well plate, and to another set of 7 wells we added $0.5 \mathrm{~mL}$ SDC/casein/cycloheximide in order to measure baseline pathway output. We sonicated $1.0 \mathrm{~mL}$ of each culture to disperse cell clumps and added $50 \mu \mathrm{L}$ of each culture to the wells containing SDC/casein/cycloheximide and the pheromone-dilution series. We incubated the plate at $30^{\circ} \mathrm{C}$ for 3 hours, shaking at $300 \mathrm{rpm}$, at which point we stopped the experiment and allowed the fluorophores to mature as described above. We sonicated the plate and quantified reporter fluorescence by microscopy (3.1.a) and flow cytometry. 5 ul of cells was subjected to flow cytometric analysis using a BD LSR-II flow cytometer (UCSF QB3 core facility) equipped with a high throughput sampler, a $488 \mathrm{~nm} 100 \mathrm{~mW}$ laser, FITC emission filter and FACS DIVA software to compile .fcs files.

We compared pathway output in RCY1130pch, RCY2005pch, DPY1003, TCY3328, TCY3329, and TCY3330. Starting from single colonies, we inoculated $4 \mathrm{~mL}$ cultures in SDC (RCY1130pch), SD/-W (RCY2005pch), or SD/-U (DPY1003, TCY3328, TCY3329, and TCY3330) and grew over the course of the day to obtain log phase cells. We diluted overnight $4 \mathrm{~mL}$ cultures in the same media to obtain mid-log phase cells in the morning, at which point we adjusted to $\mathrm{OD}_{600}=0.25$ and allowed the cells to grow for one generation (90 minutes). We prepared a 4 -fold dilution series of pheromone in SDC/casein ranging from $20 \mathrm{nM}-0.31 \mathrm{nM}$. We aliquoted $6 \times 0.5 \mathrm{~mL}$ of each dilution into a 96-well deep well plate, and to another set of 6 wells we added $0.5 \mathrm{~mL} \mathrm{SDC/casein/cycloheximide} \mathrm{in} \mathrm{order} \mathrm{to} \mathrm{measure} \mathrm{baseline}$ pathway output. We sonicated $1.0 \mathrm{~mL}$ of each culture to disperse cell clumps and added $50 \mu \mathrm{L}$ of each culture to the wells containing SDC/casein/cycloheximide and the pheromone-dilution series. We incubated the plate at $30^{\circ} \mathrm{C}$ for 3 hours, shaking at $300 \mathrm{rpm}$, at which point we stopped the experiment and allowed the fluorophores to mature as described above. We sonicated the plate and quantified reporter fluorescence by microscopy (3.1.a).

We compared pathway output in TCY3154, DPY250, DPY5001, TCY3344.2, and TCY3345.2. Starting from single colonies, we inoculated $4 \mathrm{~mL}$ cultures in SD/-W (TYC3154 and DPY250), SD/-W-U (DPY5001, TCY3344.2, and TCY3345.2) and grew over the course of the day to obtain log phase cells. We diluted overnight $4 \mathrm{~mL}$ cultures in the same media to obtain mid$\log$ phase cells in the morning, at which point we adjusted to $\mathrm{OD}_{600}=0.25$ and allowed the cells to grow for one generation (90 minutes). We prepared a 4-fold dilution series of pheromone in $\mathrm{SDC}$ /casein ranging from $20 \mathrm{nM}-0.31 \mathrm{nM}$. We aliquoted $5 \times 0.5 \mathrm{~mL}$ of each dilution into a 96 -well deep well plate, and to another set of 5 wells we added $0.5 \mathrm{~mL}$ SDC/casein/cycloheximide in order to measure baseline pathway output. We sonicated $1.0 \mathrm{~mL}$ of each culture to disperse cell clumps and added $50 \mu \mathrm{L}$ of each culture to the wells containing SDC/casein/cycloheximide and the pheromone-dilution series. We incubated the plate at $30^{\circ} \mathrm{C}$ for 3 hours, shaking at $300 \mathrm{rpm}$, at which point we stopped the experiment and allowed the fluorophores to mature as described above. We sonicated the plate and quantified reporter fluorescence by microscopy (3.1.a).

\section{Bioinformatic analysis}

Previously determined sites of phosphorylation, protein domains, protein/protein interaction information and gene and protein sequences were obtained from primary literature, the Biobase YPD database [www.proteome.com] and the Saccharomyces genome database (S288C strain background) [www.yeastgenome. org].

We used tree-assisted ortholog alignments to identify $S$. cerevisiae pheromone pathway protein orthologs in other yeast species. In two cases (Fus3 and Kss1) orthologs from some species were not included in the ortholog assignments. In these cases we obtained the absent ortholog sequences from the $Y$. lipolytica database (9) from orthlogues assigned in (10) and from the Wapinski update (January 2009) that is available on SGD (yeastgenome.org) in the fungal orthogroups repository. We used the Yeast Gene Order 
Browser (YGOB) to distinguish orthologs and paralogs in cases of retained duplicates (11).

We aligned regions of S. cerevisiae Ste12, Dig1, and Ste50 to the best matching sequences in the orthologs identified by Wapinski et al. to demonstrate the conservation of motifs and phosphorylation sites.

\section{Computational modeling}

For each protein of interest (Ste12, Dig1, Ste50), we wrote a set of ordinary differential equations (ODEs) to describe roles for phosphorylation sites in the context of pheromone signaling. All reaction equations are based on mass action kinetics, and a Hill function describes the transcriptional induction step. Although the models employ the standard mathematical approach of ODEbased mass action equations, the models qualitatively differ from standard approaches in two important ways.

First, instead of explicitly keeping track of concentrations of the species in physical units, the models represent the species in abstract, non-physical units of "activity". These abstract species still exert their effects in proportion to their amounts, and thus still operate in a mass action paradigm. This method emphasizes the generic architectural constraints of the pathway and avoids the requirement of fitting specific unmeasured parameters.

Second, the models incorporate phosphorylation in a noncanonical way. Standard ODE models represent unmodified and modified proteins as distinct species that have their own production, decay and reaction rates. Here, in contrast, phosphorylation does not generate novel species with new properties, rather phosphorylation tunes the inherent activity of the native proteins by acting "in trans". In this way, the models remain uncluttered by additional unmeasured parameters and assumptions, and therefore emphasize how the phosphorylation events tune the activity of the protein they modify. This approach could be used to study the role of any protein modification.

To implement the models, equations were coded and simulated across a pheromone dose response in MatLab R2008a using the ODE23s solver (code included as supplementary material). Parameters were all set to 1 to initiate the system, and were modified to fit the experimental data. Once a set of parameters was obtained that fit the experimental data, we performed sensitivity analysis by sweeping through 2- and 10-fold increases and decreases of each parameter and simulating the ratio of mutant to reference strain transcription in response to $20 \mathrm{nM}$ pheromone (supplementary figures). The observed differences between the mutants and reference strain transcriptional responses were insensitive to most parameter changes. Sensitive parameters are discussed in the main text.

\section{Supporting Information}

Figure S1 Ste12 ${ }^{\mathrm{S402A}}$ and Ste $12^{\mathrm{T406A}}$ have the same dose response as wild type. Cells bearing wild type or mutant Ste 12 were treated with the indicated doses of pheromone and PRM1 driven mCherry was measured by flow cytometry. (PDF)

Figure S2 Modeling phosphorylation sites in trans. The modeling strategy employed here treats the phosphorylation sites as separate entities from the proteins they modify (left). These phosphorylation sites ( $p$ sites) activate or inhibit the protein they modify (substrate) as a function of their concentration. In the abstracted example shown, reminiscent of the S202-mediated negative feedback loop, the effect of the phosphorylation site is the same whether "in trans" or as a "new species" - that is, a net negative effect on the activity of the MAPK. Advantages of the "in trans" model are that there is one fewer species and 4 fewer reaction rates to model. Moreover, there are fewer mechanistic assumptions in the "in trans" model, since the net inhibitory effect is all that is modeled. In the "new species" model, a mechanism must be specified for how the inhibitory effect happens (here it is shown as increasing the rate of MAPK dephosphorylation). The "in trans" formulation allows the models to be simplified, generic and easily modifiable without making mechanistic assumptions. (PDF)

Figure S3 Results of Ste12 modeling are robust to changes in parameters. The strength of each parameter depicted in the cartoon model as well as the degradation constant for Ste 12 were increased and decreased by 2-fold (top panels) and 10 -fold (bottom panels), and the ratio of simulated output of wild type to $\mathrm{S} 400 \mathrm{~A}$ at $20 \mathrm{nM}$ pheromone (the mutant phenotype) is plotted. The phenotype persists in the presence of 2-fold changes, but changes in magnitude with 10 -fold changes to the parameters. (PDF)

Figure S4 Dose responses of Ste50 ${ }^{\mathrm{S202A}, \mathrm{T205A}}$ and Ste50 ${ }^{\text {T205A }}$. Cells bearing mutant versions of Ste50 were treated with pheromone in the presence of 1-NM-PP1, imaged by epifluorescent microscopy and quantified using Cell-ID.

(PDF)

Figure S5 Results of Ste50 modeling are robust to changes in parameters. The strength of each parameter depicted in the cartoon model were increased and decreased by 2 fold (top panels) and 10-fold (bottom panels), and the ratio of simulated output of wild type to S202A at $20 \mathrm{nM}$ pheromone (the mutant phenotype) is plotted. The phenotype persists in the presence of 2-fold and 10-fold changes.

(PDF)

Figure S6 Dig1 ${ }^{\text {T277A,S279A,T280A }}$ resides in the nucleus and does not destabilize Ste12. Cells bearing CFP-Ste12 and either wild type or mutant Dig1-YFP were imaged in by epifluorescence microscopy. Single channel and merged images are shown.

(PDF)

Figure S7 Dig1 ${ }^{\text {S269A,S272A,S275A }}$ activates the pheromone response like wild type. A. Alignment of the full tryptic peptide identified by mass spec as being phosphorylated against Digl orthologs in other yeast species. B. Cells bearing Dig1, Digl ${ }^{\text {S269A,S272A,S275A }}$,

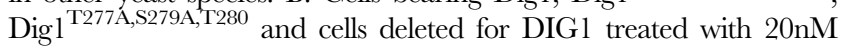
pheromone for $3 \mathrm{hrs}$. Digl $\mathrm{S}^{\mathrm{S} 69 \mathrm{~A}, \mathrm{~S} 272 \mathrm{~A}, \mathrm{~S} 275 \mathrm{~A}}$ is indistinguishable from wild type.

(PDF)

Figure S8 Dig1 model 2 and model 4 do not fit the experimental data. A. Cartoon depiction of Digl model 2, in which phosphorylation of T277 and T280 is constitutive and independent of pheromone. In this model, in order to have a dose dependent increase in Digl activity in the presence of constitutive phosphorylation of T277 and T280, we inserted a pheromone bypass directly on Dig1. B. Simulation of Digl model 2: To agree with the wild type response, the strength of this bypass was such that the contribution of phospho-T277 and -280 were negligible. Thus the mutants are identical to the wild type. C. Cartoon depiction of Digl model 4, in which dephosphorylation of both T277 and T280 is pheromone-dependent. D. Simulation of Digl model 4: The strength of the influence of each phosphorylation site had to be so strong, such that if one site was mutated, the effect of the other dominated over the pheromone response. Thus, the mutants cannot recapitulate the experimental dose responses. (PDF) 
Figure S9 Dig1 model 1 is robust to 2 -fold changes in parameters that determine the influence of the phosphorylation sites. The strength of each parameter depicted in the cartoon model were increased and decreased by 2-fold (top panels) and 10-fold (bottom panels), and the ratios of simulated output of wild type to T277A (black) and wild type to T280A (red) at $20 \mathrm{nM}$ pheromone are plotted. The T277A phenotype persists (within pink boxes) with 2-fold changes to every parameter. The T280A phenotype persists except when the strength of the the Digl-independent pheromone bypass (B4) becomes too strong. 10fold changes to any parameter significantly change the simulation results.

(PDF)

Figure S10 Dig1 model 3 is robust to 2-fold changes in parameters that determine the influence of the phosphorylation sites. The strength of each parameter depicted in the cartoon model were increased and decreased by 2-fold (top panels) and 10-fold (bottom panels), and the ratios of simulated output of wild type to T277A (black) and wild type to T280A (red) at $20 \mathrm{nM}$ pheromone are plotted. The T277A phenotype persists (within pink boxes) with 2-fold changes to every parameter. The T280A phenotype persists except when the strength of the the Digl-independent pheromone bypass (B5) becomes too strong. 10fold changes to any parameter significantly change the simulation results.

(PDF)

Table S1 Strains used in this study. (DOCX)

\section{References}

1. Bublil EM, Yarden Y (2007) The EGF receptor family: spearheading a merger of signaling and therapeutics. Current opinion in cell biology 19: 124-134.

2. Ferrell JE Jr, Machleder EM (1998) The biochemical basis of an all-or-none cell fate switch in Xenopus oocytes. Science 280: 895-898.

3. Hubbard SR, Miller WT (2007) Receptor tyrosine kinases: mechanisms of activation and signaling. Current opinion in cell biology 19: 117-123.

4. Knoblich JA (2010) Asymmetric cell division: recent developments and their implications for tumour biology. Nature reviews Molecular cell biology 11: 849860 .

5. Weston CR, Davis RJ (2007) The JNK signal transduction pathway. Current opinion in cell biology 19: 142-149.

6. Pawson T, Scott JD (2005) Protein phosphorylation in signaling--50 years and counting. Trends Biochem Sci 30: 286-290.

7. Stroud R (1991) Mechanisms of biological control by phosphorylation. Current opinion in structural biology 1: 826-835

8. Errede B, Gartner A, Zhou Z, Nasmyth K, Ammerer G (1993) MAP kinaserelated FUS3 from S. cerevisiae is activated by STE7 in vitro. Nature 362: 261264.

9. Gartner A, Nasmyth K, Ammerer G (1992) Signal transduction in Saccharomyces cerevisiae requires tyrosine and threonine phosphorylation of FUS3 and KSS1. Genes Dev 6: 1280-1292.

10. Gorner W, Durchschlag E, Martinez-Pastor MT, Estruch F, Ammerer G, et al. (1998) Nuclear localization of the C2H2 zinc finger protein Msn2p is regulated by stress and protein kinase A activity. Genes Dev 12: 586-597.

11. Verma R, Annan RS, Huddleston MJ, Carr SA, Reynard G, et al. (1997) Phosphorylation of Sic lp by G1 Cdk required for its degradation and entry into S phase. Science 278: 455-460.

12. Colman-Lerner A, Gordon A, Serra E, Chin T, Resnekov O, et al. (2005) Regulated cell-to-cell variation in a cell-fate decision system. Nature 437: 699706 .

13. Yu RG, Pesce CG, Colman-Lerner A, Lok L, Pincus D, et al. (2008) Negative feedback that improves information transmission in yeast signalling. Nature 456: $755-761$.

14. Gordon A, Colman-Lerner A, Chin TE, Benjamin KR, Yu RC, et al. (2007) Single-cell quantification of molecules and rates using open-source microscopebased cytometry. Nat Methods 4: 175-181.

15. Dohlman HG, Thorner JW (2001) Regulation of G protein-initiated signal transduction in yeast: paradigms and principles. Annu Rev Biochem 70: 703754.

16. Ramezani-Rad M (2003) The role of adaptor protein Ste50-dependent regulation of the MAPKKK Ste11 in multiple signalling pathways of yeast. Curr Genet 43: 161-170.
Table S2 Primers used for strain construction and mutagenesis.

(DOCX)

Table S3 Plasmids used in this study.

(DOCX)

Table S4 Public database links to phosphopeptides analyzed in this study.

(DOCX)

Table S5 P-values for mutant phenotypes.

(DOCX)

Materials and Methods S1.

(DOCX)

Text S1 Matlab code for simulations.

(DOCX)

\section{Acknowledgments}

The authors gratefully acknowledge contributions from Richard Yu, Gustavo Pesce, Kirsten Benjamin, Annie Tsong and Ian Burbulis for scientific discussions, reagents and/or comments on the manuscript. The authors also thank Robert Maxwell for scientific discussions and input into the selection of the 4 tryptic peptides that were analyzed in this study.

\section{Author Contributions}

Conceived and designed the experiments: DP RDS RB OR. Performed the experiments: DP CR. Analyzed the data: DP CR RB OR. Contributed reagents/materials/analysis tools: RDS. Wrote the paper: DP RB OR.

17. Wu C, Leberer E, Thomas DY, Whiteway M (1999) Functional characterization of the interaction of Ste50p with Stellp MAPKKK in Saccharomyces cerevisiae. Mol Biol Cell 10: 2425-2440.

18. Good M, Tang G, Singleton J, Remenyi A, Lim WA (2009) The Ste5 scaffold directs mating signaling by catalytically unlocking the Fus3 MAP kinase for activation. Cell 136: 1085-1097.

19. Neiman AM, Herskowitz I (1994) Reconstitution of a yeast protein kinase cascade in vitro: activation of the yeast MEK homologue STE7 by STE11. Proc Natl Acad Sci U S A 91: 3398-3402.

20. Chou S, Lane S, Liu H (2006) Regulation of mating and filamentation genes by two distinct Ste12 complexes in Saccharomyces cerevisiae. Mol Cell Biol 26: 4794-4805.

21. Tedford K, Kim S, Sa D, Stevens K, Tyers M (1997) Regulation of the mating pheromone and invasive growth responses in yeast by two MAP kinase substrates. Curr Biol 7: 228-238.

22. Brent R (2009) Cell signaling: what is the signal and what information does it carry? FEBS letters 583: 4019-4024.

23. Thomson TM, Benjamin KR, Bush A, Love T, Pincus D, et al. (2011) Scaffold number in yeast signaling system sets tradeoff between system output and dynamic range. Proceedings of the National Academy of Sciences of the United States of America 108: 20265-20270.

24. Paliwal S, Iglesias PA, Campbell K, Hilioti Z, Groisman A, et al. (2007) MAPKmediated bimodal gene expression and adaptive gradient sensing in yeast. Nature 446: 46-51.

25. Malleshaiah MK, Shahrezaei V, Swain PS, Michnick SW (2010) The scaffold protein Ste5 directly controls a switch-like mating decision in yeast. Nature 465: $101-105$.

26. Letunic I, Doerks T, Bork P (2012) SMART 7: recent updates to the protein domain annotation resource. Nucleic Acids Res 40: D302-305.

27. Cherry JM, Hong EL, Amundsen C, Balakrishnan R, Binkley G, et al. (2012) Saccharomyces Genome Database: the genomics resource of budding yeast. Nucleic Acids Res 40: D700-705.

28. Ponting CP (1995) SAM: a novel motif in yeast sterile and Drosophila polyhomeotic proteins. Protein Sci 4: 1928-1930.

29. Truckses DM, Bloomekatz JE, Thorner J (2006) The RA domain of Ste50 adaptor protein is required for delivery of Stell to the plasma membrane in the filamentous growth signaling pathway of the yeast Saccharomyces cerevisiae. Mol Cell Biol 26: 912-928.

30. Yuan YL, Fields S (1991) Properties of the DNA-binding domain of the Saccharomyces cerevisiae STE12 protein. Mol Cell Biol 11: 5910-5918.

31. Pincus D, Chevalier MW, Aragon T, van Anken E, Vidal SE, et al. (2010) BiP binding to the ER-stress sensor Irel tunes the homeostatic behavior of the unfolded protein response. PLoS Biol 8: e1000415. 
32. Bodenmiller B, Campbell D, Gerrits B, Lam H, Jovanovic M, et al. (2008) PhosphoPep--a database of protein phosphorylation sites in model organisms. Nat Biotechnol 26: 1339-1340.

33. Stark C, Su TC, Breitkreutz A, Lourenco P, Dahabieh M, et al. (2010) PhosphoGRID: a database of experimentally verified in vivo protein phosphorylation sites from the budding yeast Saccharomyces cerevisiae. Database (Oxford) 2010: bap026.

34. Gnad F, Gunawardena J, Mann M (2011) PHOSIDA 2011: the posttranslational modification database. Nucleic Acids Res 39: D253-260.

35. Gruhler A, Olsen JV, Mohammed S, Mortensen P, Faergeman NJ, et al. (2005) Quantitative phosphoproteomics applied to the yeast pheromone signaling pathway. Mol Cell Proteomics 4: 310-327.

36. Pincus D, Benjamin K, Burbulis I, Tsong AE, Resnekov O (2010) Reagents for investigating MAPK signalling in model yeast species. Yeast 27: 423-430.

37. Heiman MG, Walter P (2000) Prmlp, a pheromone-regulated multispanning membrane protein, facilitates plasma membrane fusion during yeast mating. J Cell Biol 151: 719-730.

38. Sneddon MW, Faeder JR, Emonet T (2011) Efficient modeling, simulation and coarse-graining of biological complexity with NFsim. Nat Methods 8: 177-183.

39. Linding R, Jensen LJ, Pasculescu A, Olhovsky M, Colwill K, et al. (2008) NetworKIN: a resource for exploring cellular phosphorylation networks. Nucleic Acids Res 36: D695-699.

40. Ingrell CR, Miller ML, Jensen ON, Blom N (2007) NetPhosYeast: prediction of protein phosphorylation sites in yeast. Bioinformatics 23: 895-897.

41. Chernomoretz A, Bush A, Yu R, Gordon A, Colman-Lerner A (2008) Using Cell-ID 1.4 with $\mathrm{R}$ for microscope-based cytometry. Curr Protoc Mol Biol Chapter 14: Unit 1418.

42. Bishop AC, Ubersax JA, Petsch DT, Matheos DP, Gray NS, et al (2000) A chemical switch for inhibitor-sensitive alleles of any protein kinase. Nature 407: 395-401.

43. Olson KA, Nelson C, Tai G, Hung W, Yong C, et al. (2000) Two regulators of Ste12p inhibit pheromone-responsive transcription by separate mechanisms. Mol Cell Biol 20: 4199-4209.
44. McCullagh E, Seshan A, El-Samad H, Madhani HD (2010) Coordinate control of gene expression noise and interchromosomal interactions in a MAP kinase pathway. Nature cell biology 12: 954-962.

45. Houser JR, Ford E, Nagiec MJ, Errede B, Elston TC (2012) Positive roles for negative regulators in the mating response of yeast. Molecular systems biology 8 : 586.

46. Wood AJ, Lo TW, Zeitler B, Pickle CS, Ralston EJ, et al. (2011) Targeted genome editing across species using ZFNs and TALENs. Science 333: 307.

47. Nagiec MJ, Dohlman HG (2012) Checkpoints in a yeast differentiation pathway coordinate signaling during hyperosmotic stress. PLoS genetics 8: e1002437.

48. Shock TR, Thompson J, Yates JR 3rd, Madhani HD (2009) Hogl mitogenactivated protein kinase (MAPK) interrupts signal transduction between the Kss1 MAPK and the Tecl transcription factor to maintain pathway specificity. Eukaryotic cell 8: 606-616.

49. Yamamoto K, Tatebayashi K, Tanaka K, Saito H (2010) Dynamic control of yeast MAP kinase network by induced association and dissociation between the Ste50 scaffold and the Opy2 membrane anchor. Molecular cell 40: 87-98.

50. Brown CJ, Johnson AK, Dunker AK, Daughdrill GW (2011) Evolution and disorder. Current opinion in structural biology 21: 441-446.

51. Holt LJ, Tuch BB, Villen J, Johnson AD, Gygi SP, et al. (2009) Global analysis of Cdk1 substrate phosphorylation sites provides insights into evolution. Science 325: $1682-1686$

52. Iakoucheva LM, Radivojac P, Brown CJ, O’Connor TR, Sikes JG, et al. (2004) The importance of intrinsic disorder for protein phosphorylation. Nucleic acids research 32: 1037-1049.

53. Radivojac P, Iakoucheva LM, Oldfield CJ, Obradovic Z, Uversky VN, et al. (2007) Intrinsic disorder and functional proteomics. Biophysical journal 92: 1439-1456.

54. Wright PE, Dyson HJ (1999) Intrinsically unstructured proteins: re-assessing the protein structure-function paradigm. Journal of molecular biology 293: 321-331. 